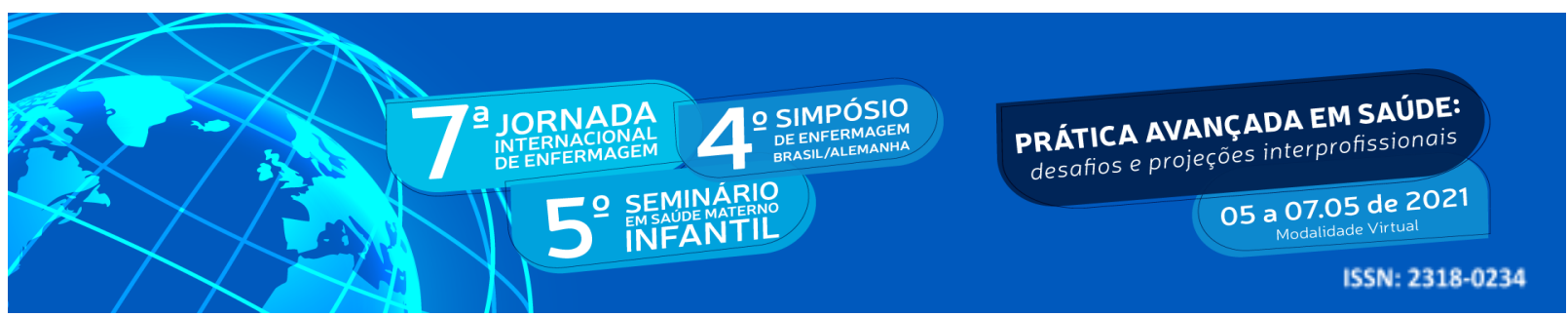

DOI: http://doi.org/10.48195/jie2021-151

\title{
ATUALIZAÇÃO PROFISSIONAL: FERRAMENTA INDISPENSÁVEL PARA O ÊXITO DOS SERVIÇOS DE PROMOÇÃO DA SAÚDE
}

\author{
Gicelle Moraes Martelli ${ }^{1}$; Dielli Arend Teixeira ${ }^{2}$; Graziele Alves Flores ${ }^{4}$; Pabline Pivetta \\ de Oliveira ${ }^{5}$;Ticiana Aita Xavier ${ }^{6}$; Regina Gema Santini Costenaro ${ }^{7}$
}

\begin{abstract}
RESUMO
O presente artigo tem como objetivo fundamentar as pesquisas científicas que abordam acerca das ferramentas que contribuem no êxito dos serviços de promoção a saúde. Trata-se de um estudo descritivo do tipo revisão de literatura, definida como método de elaboração narrativa. As buscas ocorreram pelas bases de dados da Biblioteca Virtual em Saúde (BVS) e Scientific Electronic Library Online (Scielo). Com base na síntese e análise dos artigos, construiu-se dois eixos temáticos como guia de discussão denominados: Cuidados ao Recém-nascido e Educação Permanente em Saúde. A partir do levantado, foi possível observar a evolução das técnicas e o fortalecimento durante a graduação, somado ao conhecimento constante de novos estudos. Dessa maneira, contribui para uma renovação de ideias e técnicas de procedimentos que aprimoram o trabalho em equipe. Além disso, as boas práticas contribuem para promoção de saúde e prevenção de agravos.
\end{abstract}

Palavras-chave: Cuidado de Enfermagem; Recém-nascido; Educação Continuada.

\begin{abstract}
This article aims to support scientific research that addresses the tools that contribute to the success of health promotion service. This is a descriptive study of the literature review type, defined as a method of narrative elaboration. The searches took place through the databases of the Virtual Health Library (VHL) and Scientific Electronic Library Online (Scielo). Based on the synthesis and analysis of the articles, two thematic axes were constructed as a discussion guide called: Newborn Care and Continuing Health Education. From the survey, it was possible to observe the evolution of the techniques and the strengthening during graduation, added to the constant knowledge of new studies. Thus, it contributes to a renewal of ideas and techniques of procedures that improve teamwork. In addition, good practices contribute to health promotion and disease prevention.
\end{abstract}

Key Words: Nursing Care; Newborn; Continuing Education.

\footnotetext{
1 Estudante do Curso de Enfermagem. Bolsista voluntária PROBIC. Universidade Franciscana. E-mail: gicelle1912@gmail.com

${ }^{2}$ Estudante. Curso de Enfermagem da Universidade Franciscana - UFN. E-mail: dielliarend@gmail.com

${ }^{3}$ Estudante. Curso de Enfermagem. Bolsista FAPERGS. UFN. E-mail: flores.graziele8@gmail.com

${ }^{4}$ Estudante do Curso de Enfermagem. Bolsista PROBIC. UFN. E-mail: pablinepivetta@gmail.com

${ }^{5}$ Médica pediatra. Aluna do mestrado profissional em saúde materna e infantil. Universidade Franciscana. UFN.

E-mail: tici xavier@hotmail.com

${ }^{6}$ Orientador. Professor doutor. Universidade Franciscana-UFN. E-mail: regina@ufn.edu.br
} 


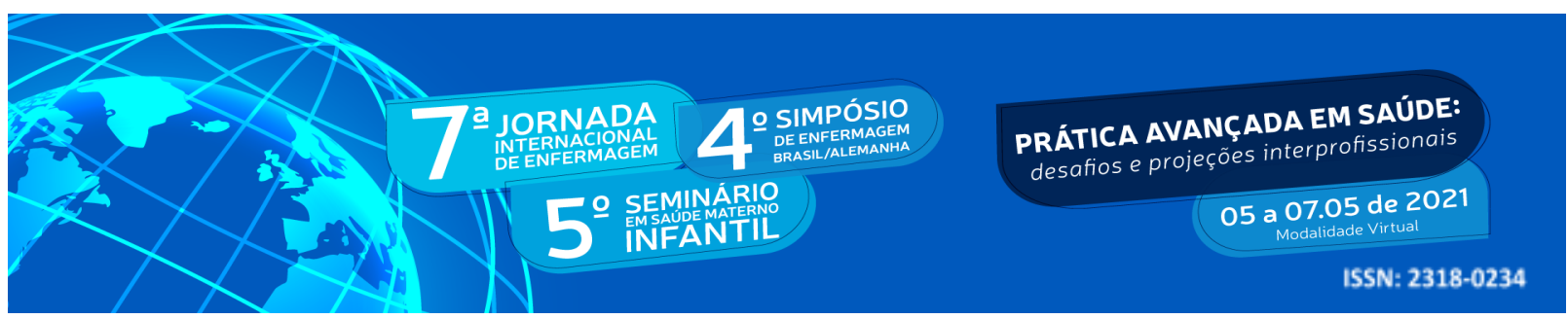

\section{INTRODUÇÃO}

O ser humano, durante toda sua existência, mantém-se em constante processo de aprendizagem e atualização nos diferentes aspectos que permeiam a vida humana, seja no âmbito profissional, cultural ou também nas notícias de acontecimentos diários. No aspecto profissional, salienta-se a área da saúde que está em constante processo de adequação com relação às condutas terapêuticas, meios diagnósticos, surgimento de novas medicações, dentre outras atividades cotidianas.

Esta necessidade de atualização é sentida e manifestada pelos profissionais nas instituições de saúde, uma vez que estas, apresentam espaços privilegiados para a aquisição de habilidades, realização de reflexões que possam contribuir com a construção do conhecimento. Nessa perspectiva, a Educação Permanente em Saúde - EPS pode atender estas demandas à medida que oferece novas possibilidades para transformar as práticas profissionais dos trabalhadores de saúde e consequentemente, também pode contribuir para a disposição dos processos de trabalho (SILVA, SANTOS, BARBOSA, 2017).

A Política Nacional de Educação Permanente em Saúde (PNEPS) é uma ação estruturante do Departamento de Gestão da Educação na Saúde e da Secretaria de Gestão do Trabalho. A qual foi fomentada pela portaria GM/MS de $n^{0} 198$, de 13 de fevereiro de 2004. Em 2007, passou por alterações, envolvendo as novas diretrizes e estratégias da PNEPS. Dessa maneira, percebe-se que a transformação nos serviços, na educação/ensino e na administração do sistema de saúde não pode estar relacionada a uma simples questão técnica, pois abrange, mudança em três aspectos, quais sejam, nas relações, nos processos de saúde e nas pessoas. Assim, deve ocorrer um trabalho articulado entre o sistema de saúde, em suas esferas de gestão e as instituições formadoras, objetivando desenvolver e promover uma saúde que esteja relacionada às necessidades dos seus usuários, além de apreciar os atores sociais relacionados ao mundo do trabalho (Brasil, 2014).

Dentre as áreas de saúde que muito tem aprimorado e adequado seus cuidados, destaca-se a saúde materna e infantil, e desta a saúde do neonato e da criança. Na rede pública, foi criada a Rede Cegonha no ano de 2011 pelo Ministério da Saúde no âmbito do SUS, criando estratégias para ampliar o cuidado e assegurar o direito ao planejamento 


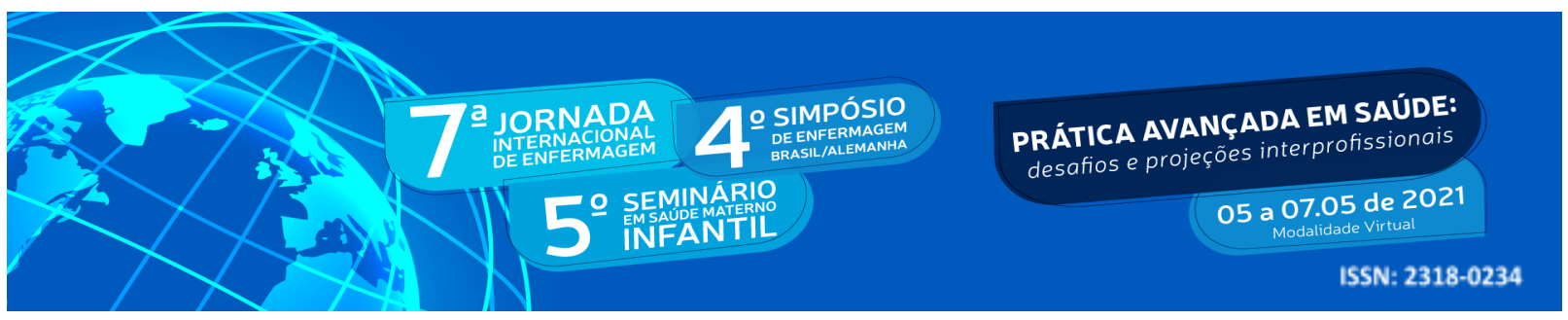

reprodutivo, atenção humanizada durante a gestação, parto e período do puerpério. Assim, as crianças também seriam asseguradas em relação ao nascimento saudável e seguro, bem como um crescimento e desenvolvimento saudável (MARQUES, 2015).

Os recém-nascidos possuem suas singularidades, as quais resultam em uma instabilidade dos sistemas hormonais e neurológicos no processo de desenvolvimento e adaptação, em decorrência da imaturidade dos diferentes órgãos. Ademais, é imprescindível reconhecer a comunicação não-verbal emitida pelo RN, para assim, conseguir atender suas necessidades de cuidado. Os bebês são influenciados pelos ambientes em que estão inseridos, como sons, movimentos e gestos das pessoas que estão na sua rotina. Esses estímulos, quando bem aplicados, promovem um bom desempenho cognitivo e afetivo ao bebê (BARREIROS, et al., 2021).

A equipe de enfermagem, deve dar assistência no processo de trabalho de parto. Necessita de recursos e materiais para um amparo eficaz. É indispensável que a equipe multiprofissional esteja preparada com uma sala de parto com aspirador, compressas e lençóis esterilizados, além, de materiais para episiotomia (em casos de partos vaginais), aspirador e berço aquecido (BARREIROS, et al., 2021).

Outrossim, a Rede Cegonha ampara a mulher com consultas de pré-natal de qualidade, sendo acolhida e avaliada para a classificação de risco e de vulnerabilidade. $\mathrm{O}$ vínculo entre a equipe e a gestante, é muito importante para tornar a rede efetiva. A gestante deve ter garantia de transporte seguro (GIOVANNI, 2013).

\section{OBJETIVO}

Objetivou-se neste estudo, conhecer e discutir sobre oque têm sido publicado sobre a promoção do conhecimento, referente as capacitações e de educação permanenteas nos serviços de cuidados com recém-nascidos na primeira semana de vida.

\section{METODOLOGIA}

Trata-se de um estudo descritivo do tipo revisão de literatura, definida como método de elaboração narrativa. As buscas ocorreram pelas bases de dados da Biblioteca Virtual em Saúde (BVS) e Scientific Electronic Library Online (Scielo). A seleção dos artigos foi 


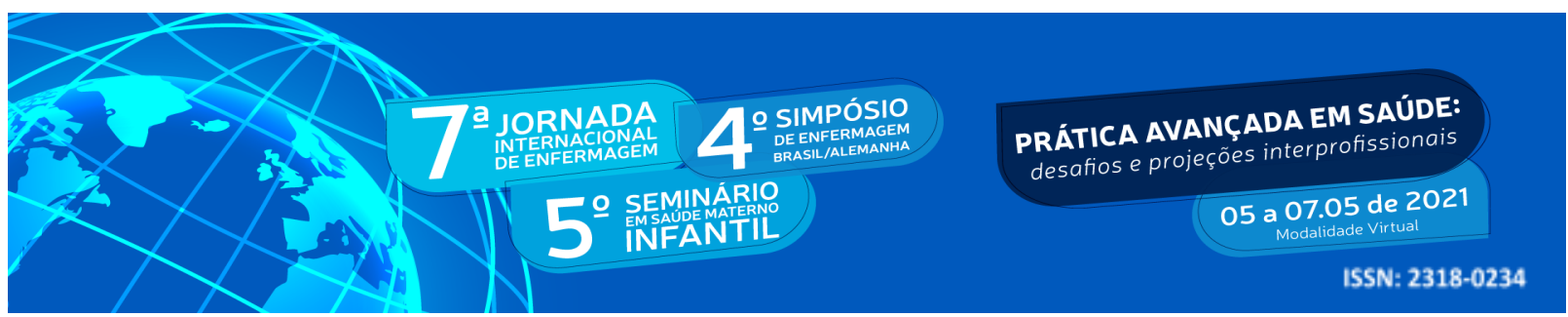

realizada no mês de março de 2021, por meio dos descritores em ciências da saúde (DesCS), "cuidados de enfermagem" and "recém-nascido" and "educação continuada".

Nesta busca totalizaram 368 artigos científicos nas bases de dados selecionadas. Onde foram aplicados os filtros, na BVS: texto completo, MEDLINE, LILACS, BDENF, idioma português, recorte temporal de 10 anos e operador booleano "And".

No primeiro momento, foi encontrado um artigo na base de dados do Scielo, que atendeu o objetivo de busca. Em um segundo momento, utilizou-se a mesma estratégia de busca na base de dados da BVS, com a utilização dos filtros citados acima, resultando em treze artigos. Entretanto, apenas cinco atenderam a temática da pesquisa. A análise sucedeu por meio da leitura do título dos estudos encontrados, resumo, assunto, e quando necessário, leitura na íntegra.

\section{RESULTADOS E DISCUSSÃO}

Com base na síntese e análise dos artigos, construiu-se dois eixos temáticos como guia de discussão denominados, conforme segue:

\subsection{Cuidados ao Recém-nascido}

O cuidado com o RN, pós nascimento, é permeado de muitas especificidades uma vez que está se habituando com avida extrauterina. A família deve estar inserida em todas as decisões durante o parto e após o nascimento do RN. A equipe deve realizar uma conversa clara e de qualidade, compartilhando informações e destacando o planejamento do cuidado, assim como, os potenciais riscos e benefícios dos procedimentos que são realizados. Evitando assim, estresse parental no hospital e no puerpério durante a internação. Os profissionais devem salientar que os responsáveis pelo $\mathrm{RN}$, devem estar integrados nos cuidados com os recém-nascidos durante a internação. Mostrando que os pais, são importantes para o cuidado e manutenção da saúde dos seus filhos (SILVA, et al. 2016).

No pensar de SOUZA (2014), o Método Mãe-Canguru, também conhecido por MMC, consiste em colocar o recém-nascido entre as mamas da mãe, para que o calor gerado pelo 


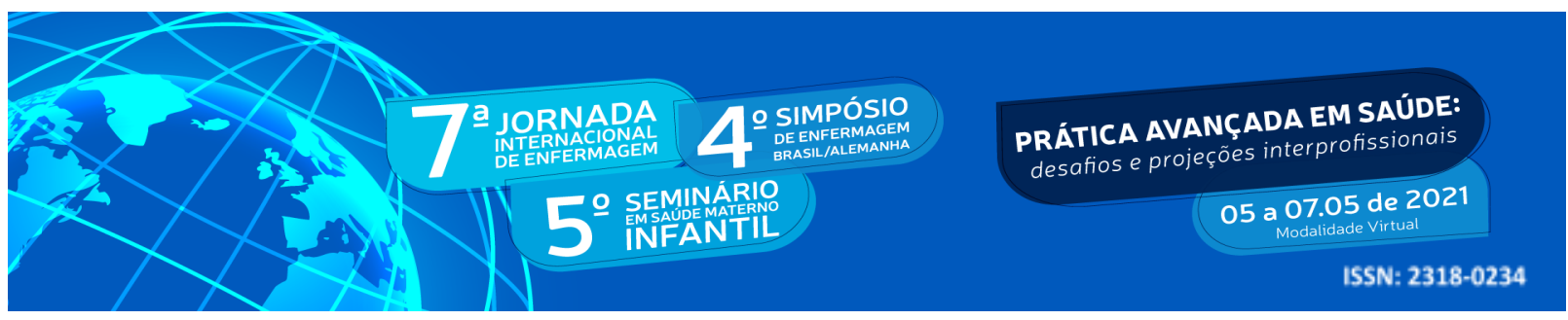

corpo da mesma, possa aquecer o RN. Essa tecnologia assistencial neonatal, do contato pele a pele contribui na para a alta hospitalar, mais rápida, evitando superlotações e infecções hospitalares. Tal método, necessita do consentimento dos pais, familiares e profissionais, visando ser um ato prazeroso para ambos. Corroborando, Santo (2011) salienta que o contato pele apele, deve ser utilizado logo após o nascimento favorecendo o acolhimento e vinculo entre a mãe com o $\mathrm{RN}$ e consequentemente fortalece o aleitamento materno exclusivo.

Estas condutas, embasaram as equipes de parto e de cuidado ao $\mathrm{RN}$, e para isso foi necessário capacitações e aprofundamento científico para tornar a assistência mais efetiva. Entretanto, ainda é falha a compreensão por alguns profissionais sobre o MMC e o contato pele a pele. É necessário, que a equipe tenha um olhar reflexivo sobre tudo que é aderido na rotina de cuidados com os recém-nascidos. Ademais, a assistência humanizada favorece o vínculo da equipe com os familiares, estimulando as mães a praticarem o método (SOUZA, et al. 2012).

Mediante estas ideias, Barreiros et al., (2021), evidencia a comunicação não-verbal emitida pelo recém-nascido. Uma vez que, o RN está inserido ao meio desconhecido, se adaptando ao novo ambiente extrauterino. Com isso o método de assistência promove o acolhimento e aconchego ao RN. O autor ressalta, assim como em Souza (2012), os índices de mortalidade e infecções hospitalares nessa fase. Os cuidados imediatos são essenciais para o desenvolvimento saudável. Com isso, a equipe deve estar preparada para identificar e intervir frente a qualquer problema que surgir e estabelecer estratégias rápidas.

Por outro lado, a equipe se torna responsável pela mãe/mulher, visto que o RN é protagonista dessa tecnologia de assistência humanizada. Assim, o contato pele a pele, resulta no bem-estar do recém-nascido, a manutenção da pressão arterial, a elevação da saturação de oxigênio e a redução da frequência respiratória. Otimizando o cuidado e acolhimento da mãe e do RN. É indispensável que a equipe insira os familiares nesse período de internação pós-parto com todos que dão entrada nas maternidades. Contribuindo para minimizar o desconhecimento do ambiente hospitalar, além dos termos técnicos normalmente utilizados pelos profissionais (SOUZA, et al. 2012).

Da mesma forma, destaca-se a importância de discutir estas condutas com os profissionais que atuam em unidades de terapia intensiva neonatais (UTIN), uma vez que este 


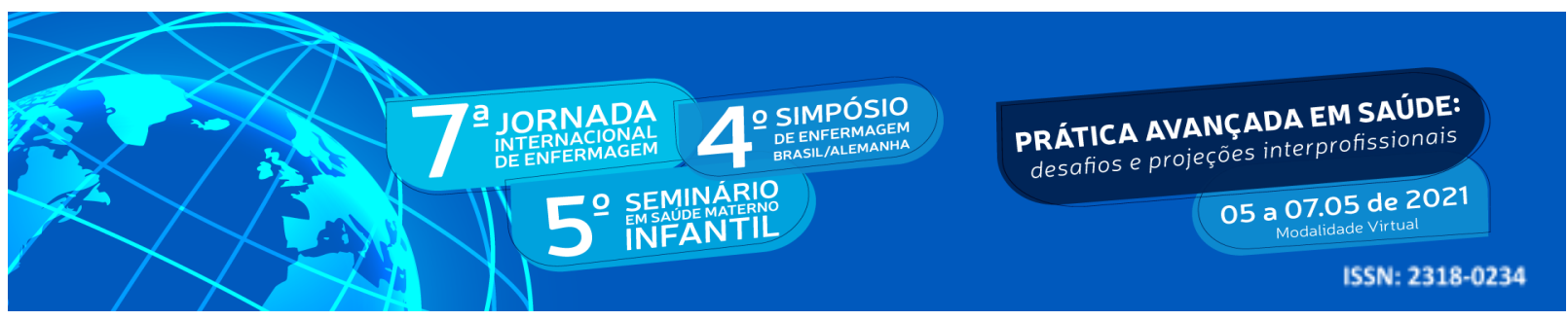

ambiente é desconhecido e o RN permanece por momentos separado da mãe, com ruídos contínuos, tornando-se estressante para o $\mathrm{RN}$, assim, é importante uma equipe multiprofissional trabalhando no desenvolvimento de um cuidado integral ao $\mathrm{RN}$, proporcionando um ambiente tranquilo com o mínimo de manipulações, oportunizando contacto pele-a-pele sempre que possível, além de atentar para o posicionamento adequado do $\mathrm{RN}$, evitando a movimentação do $\mathrm{RN}$ e consequente gastos de energias, devido ao quadro clínico (HEIDELISE, 2011).

Em contrapartida, os cuidados durante o pré-natal, também necessitam de um olhar ampliado no cenário das unidades básicas de saúde. Pesquisas mostram que a cobertura de consultas durante a gestação, foi ampliada, no entanto, no Brasil, a assistência ainda enfrenta inadequações. Dessa maneira, as equipes das Estratégias da Saúde da Família (ESF) podem intervir de forma complementar na assistência às mulheres grávidas, preparando-as para os cuidados com o $\mathrm{RN}$, reduzindo os índices de morbimortalidade neonatal. O relacionamento entre o profissional da enfermagem e a mulher colabora para que a gestante desenvolva uma confiança no trabalho de assistência do cuidado (GONÇALVES; KOWALSKI; SÁ, 2016),

As boas práticas durante o pré-natal, parto e nascimento, são garantidas pela Rede Cegonha, e exercem importantes contribuições na promoção da saúde da mãe e do RN, além de prevenir novos índices de morbidades e mortalidades. Garantindo assim, a melhoria da qualidade do atendimento e na atenção continuada da mãe e do RN (GIOVANNI, 2013).

\subsection{Educação Permanente em Saúde}

O conceito da educação permanente foi introduzido na América Latina ante a inadequação da formação profissional, que era focada exclusivamente no desenvolvimento das habilidades técnicas voltadas para o desempenho produtivo, sem a inclusão de conhecimentos, valores e de um compromisso com os aspectos políticos, éticos e sociais. Inserida no Brasil como uma proposta ético-político-pedagógica, tem como objetivo transformar e qualificar a atenção à saúde, os processos formativos, as práticas de educação em saúde, além de incentivar a organização das ações e dos serviços em uma perspectiva intersetorial (FERREIRA et al., 2019). 


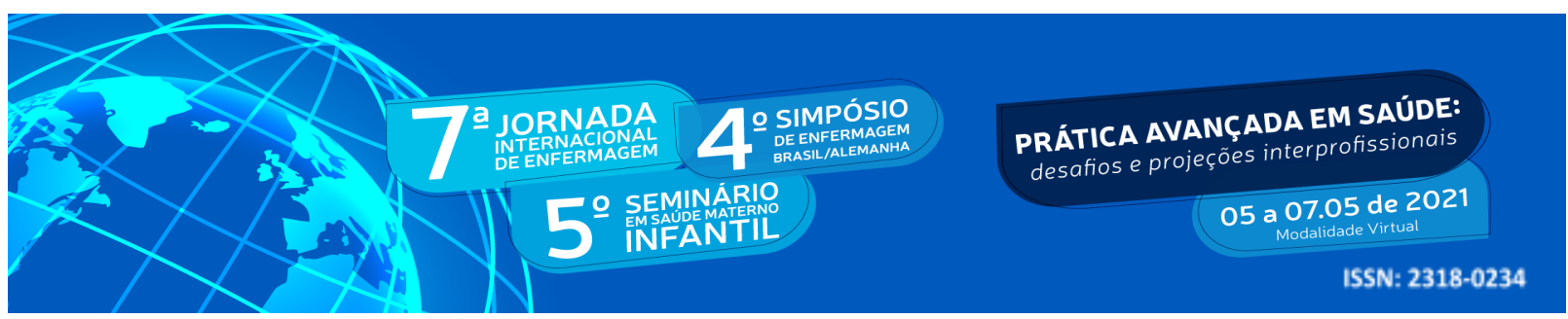

Tendo em vista estas perspectivas em campo práxis, Carvalho et al., (2015), idealiza que a educação continuada se apresenta como um processo permanente que se destina a atualizar e melhorar a capacidade profissional frente às dificuldades sociais e às evoluções técnicas e científicas uma vez que se faz necessária a atuação do enfermeiro capacitado para planejar e organizar o serviço. Com os processos de mudança que vêm ocorrendo rapidamente nos setores sociais, a saúde passa por momentos de grandes transformações, onde há a necessidade de recursos humanos que correspondam às necessidades e demandas do setor (FARIAS et al., 2017).

Dessarte, considerando o atual contexto que estamos vivenciando, acerca do isolamento pela pandemia da covid-19, Bussotti et al., (2016), afirmam positivamente acerca da construção de módulos de capacitação, com o intuito de permitir o aprendizado autônomo, privilegiando recursos de ensino interativos, concebidos de forma que o usuário possa ter a liberdade para aprender tendo em conta seus próprios ritmos e estilo de aprendizagem. Neste sentido, a educação permanente, como ferramenta de ensino tecnológico, é uma das estratégias que favorece a construção de um novo estilo na formação profissional, oportunizando aos indivíduos desenvolverem um raciocínio crítico e reflexivo dentro da área da saúde (LOPES et al., 2020). Corroborando com estas ideias, Neves et al., (2020) expressam que a utilização das tecnologias de informação, tem conquistado cada vez mais espaços como ferramentas facilitadoras para o acesso, a obtenção e transmissão do conhecimento, de maneira rápida.

Neste contexto, destaca-se a importância das relações entre os locais de trabalho e de promoção da saúde, com a formação acadêmica, e produção do conhecimento científico que contribua com a transformação da realidade, Assim. Ambos os cenários de trabalho e de formação podem desenvolver competências necessárias para aprimorar a prática profissional. Deve-se potencializar o ensino em saúde, de maneira a ser processo em movimento constante de construção, desconstrução e reconstrução, de proposições na passagem do informativo para o formativo e finalmente para o transformativo (TAVARES et al., (2016).

De tal modo em que a atualização profissional é indispensável como ferramenta na vida dos enfermeiros por meio das capacitações e promoções em saúde, também é considerada uma imprescindibilidade para estes atuantes em saúde, visto que as necessidades 


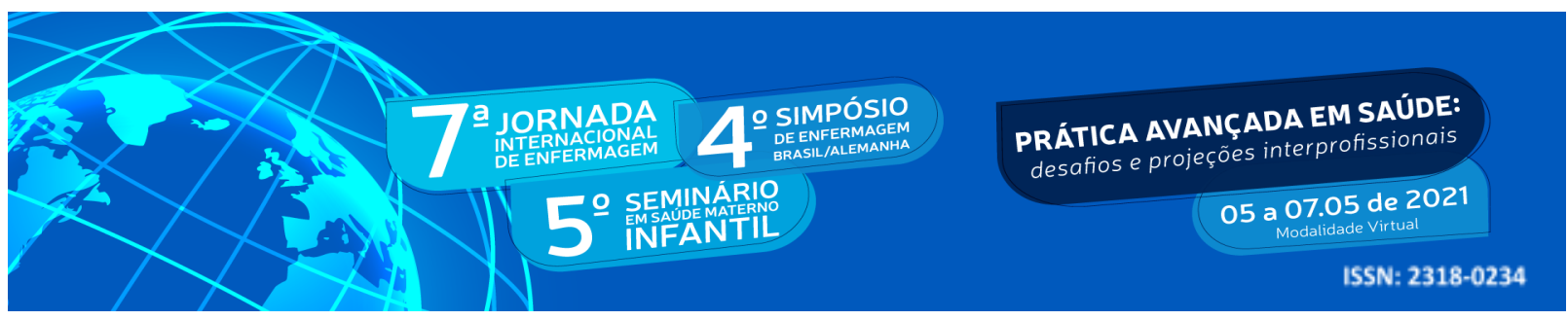

e desafios a serem superados no decorrer da trajetória profissional é baseado a partir da educação permanente em serviço ao promover a qualificação profissional (GONÇALVES; KOWALSKI; SÁ, 2016).

\section{CONCLUSÃO}

Ao efetivar uma detalhada revisão bibliográfica, se faz necessário realizar uma organização em conjunto para estabelecer estratégias e definir capacitações para a melhoria das relações e do trabalho em saúde. Além disso, o isolamento social, vivenciado atualmente, possibilitou a capacitação online, facilitando e otimizando não só o tempo, mas o posicionamento dos profissionais frente ao serviço.

A partir do levantado, foi possível observar a evolução das técnicas e o fortalecimento durante a graduação, somado ao conhecimento constante de novos estudos. Dessa maneira, contribui para uma renovação de ideias e técnicas de procedimentos que aprimoram o trabalho em equipe.

Ademais, o vínculo entre equipe e familiares dos recém-nascidos, é imprescindível para a potencializar a confiança e reduzir o tempo de internação no pós-parto. Se faz necessário um reconhecimento do ambiente pelos familiares e um acolhimento humanizado para que todos se sintam bem durante a internação hospitalar e/ou visitas. Além disso, as boas práticas de atualização nas condutas de cuidado, contribuem para promoção de saúde e prevenção de agravos, principalmente pelo fato dos acontecimentos proporcionarem um movimento constante e que por isso necessita de adequações e atualizações.

\section{REFERÊNCIAS}

BARREIROS, L. L. et al. Cuidados de enfermagem ao recém-nascido: uma revisão literária. Acesso em 27 de janeiro de 2021. Disponível em: <CUIDADOS DE ENFERMAGEM AO RECÉM-NASCIDO: UMA REVISÃO LITERÁRIA (netsaber.com.br)>

BRASIL. Ministério da Saúde. Educação Permanente em Saúde. Reconhecer a produção local de cotidianos de saúde e ativar práticas colaborativas de aprendizagem e de entrelaçamento de saberes. Brasília, 2014. 


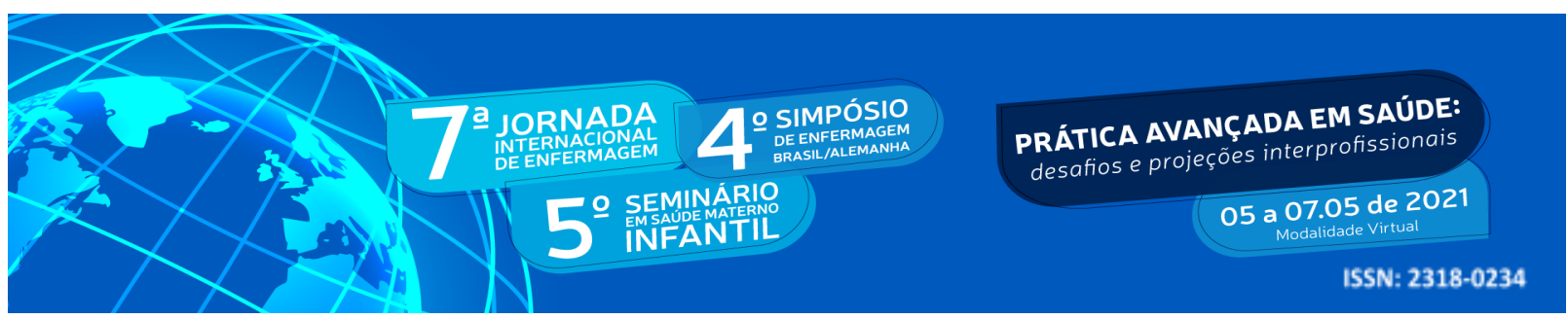

BUSSOTTI, E. A. et al. Capacitação on-line para profissionais de saúde em três regiões do Brasil. Revista Brasileira de Enfermagem, São Paulo, v. 69, n. 5, p. 981-985, jul./2016.

CARVALHO, K. X. et al. O cuidado ao recém-nascido sumetido á diálise peritoneal: desafios para a equipe de enfermagem. Cogitare Enferm, Paraná, v. 20, n. 1, p. 139-145, fev./ 2015.

SILVA, E. S.; SANTOS, C. L. R.; BARBOSA, H. L. Educação permanente em saúde no cotidiano dos serviços de saúde do sus: revisão integrativa. Revista de Inovação, Tecnologia e Ciências (RITEC), v. 3, n. 3, p. 1-8, 2017.

FARIAS, Q.L.T. et al. Implicações das tecnologias de informação e comunicação no processo de educação permanente em saúde. Reciis - Rev Eletron Comun Inf Inov Saúde. out-dez.; 11(4) 2017.

FERREIRA, L. Et al. Educação Permanente em Saúde na atenção primária: uma revisão integrativa de literatura. Saúde em Debate, Espirito Santo, v. 43, n. 120, p. 223-239, fev./ 2015.

GIOVANNI, M. REDE CEGONHA: da concepção à implantação. Brasília, 2013.

GONÇALVES, M. D.; KOWALSKI, I. S.; SÁ, A. C.; Atenção ao pré-natal de baixo risco: atitudes dos enfermeiros da estratégia saúde da família. Rev enferm UERJ,; v.24, n.6, p.18736. Rio de Janeiro, 2016.

NEVES, B. L. et al. A importância do uso do ensino a distância e das tecnologias para a capacitação dos profisssionais da saúde na atuação à COVID-19. Anais do CIET:EnPED: 2020 - São Carlos, ago. 2020.

MARQUES, C. P. C. (Org.). Redes de atenção à saúde: a Rede Cegonha. Universidade Federal do Maranhão - UFMA. Universidade Aberta do SUS - UNA-SUS São Luís, 2015.

HEIDELISE, A.L.S. Uma filosofia de cuidados. Nascer e Crescer, v.20, n.1, 2011.

SILVA, et al. Cuidado centrado na família na perspectiva de enfermeiras da Unidade de Terapia Intensiva Neonatal. Rev Rene. set-out; v.17, n.5, p. 643-50. 2016 


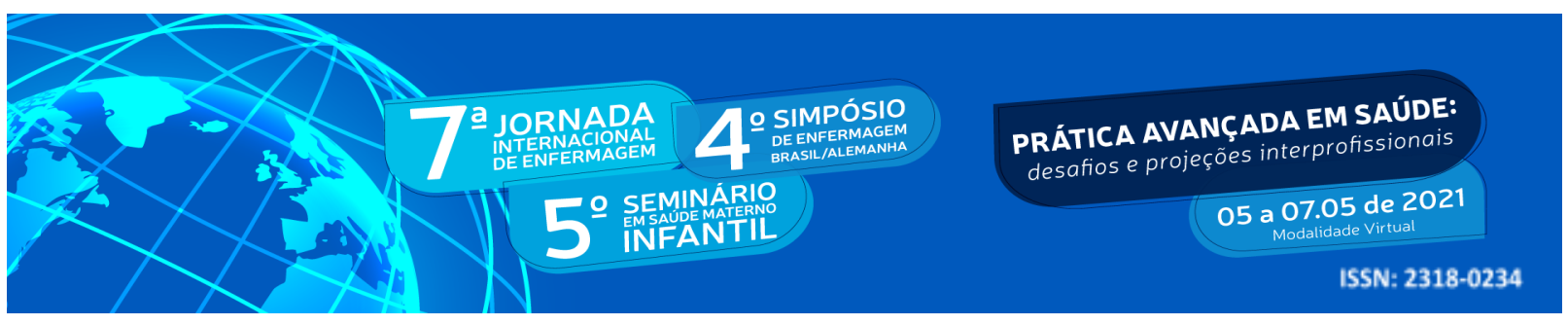

SILVA, E. S.; SANTOS, C. L. R.; BARBOSA, H. L. Educação permanente em saúde no cotidiano dos serviços de saúde do sus: revisão integrativa. Revista de Inovação, Tecnologia e Ciências (RITEC), v. 3, n. 3, p. 1-8, 2017.

SOUZA, L.P.S. et al. Método mãe-canguru: percepção da equipe de enfermagem na promoção à saúde do neonato. Rev Bras Promoç Saúde, Fortaleza, 27(3): 374-380, jul./set., 2014

TAVARES, M. D. F. et al. A promoção da saúde no ensino profissional: desafios na saúde e a necessidade de alcançar outros setores. Ciência e Saúde Coletiva, Rio de Janeiro, v. 21, n. 6, p. 1799-1808, Jun./2016. 